\title{
Genome-wide investigation of SQUAMOSA promoter binding protein-like transcription factor family in pearl millet (Pennisetum glaucum (L) R. Br.)
}

\author{
Pei Yu ${ }^{a}$, Harshraj Shinde ${ }^{b}$, Ambika Dudhate ${ }^{c}$, Daisuke Tsugama ${ }^{a}$, Shashi Kumar Gupta ${ }^{\mathrm{d}}$, \\ Shenkui Liu ${ }^{\mathrm{e}}$, Tetsuo Takano ${ }^{\mathrm{a}, *}$ \\ a Asian Natural Environmental Science Center (ANESC), The University of Tokyo, Nishitokyo-shi, Tokyo, Japan \\ ${ }^{\mathrm{b}}$ Environmental Epigenetics and Genetics Group, Department of Horticulture, College of Agriculture, Food and Environment, University of Kentucky, Lexington, KY, USA \\ ${ }^{c}$ Department of Pharmaceutical Sciences, Center for Pharmaceutical Research and Innovation, College of Pharmacy, University of Kentucky, Lexington, KY, USA \\ ${ }^{\mathrm{d}}$ International Crops Research Institute for the Semi-Arid Tropics (ICRISAT), Hyderabad, Telangana State, India \\ ${ }^{\text {e }}$ State Key Laboratory of Subtropical Silviculture, Zhejiang A and F University, Lin'an, Hangzhou, China
}

\section{A R T I C L E I N F O}

\section{Keywords:}

Pearl millet

SBP genes

Transcription factor

microRNA

Abiotic stress

\begin{abstract}
A B S T R A C T
Abiotic stress negatively affects the yield of many crops. The SQUAMOSA promoter binding protein-like proteins (SBPs) represent a family of plant-specific transcription factors which play essential roles in plant growth, development, and stress responsiveness. In this study, 18 putative SBPs (PgSBPs) were identified in the genome of pearl millet on the basis of the SBP domain. One or two zinc finger-like structure(s) and a nuclear localization signal (NLS) were found in the SBP domains of all the PgSBPs. Fourteen PgSBPs were distributed on 7 chromosomes unevenly, while the other 4 were located on the scaffolds (i.e., non-chromosomal genomic sequences). Moreover, all the PgSBPs were clustered into seven groups (I-VII) based on the phylogenetic analysis. The intron/ exon structures and the motif composition were similar between PgSBPs within the same groups. PgSBPs in groups V and VII were predicted as the targets of two microRNAs, PgmiR156q and PgmiR529b. Some PgSBPs have abscisic acid (ABA)-responsive elements and stress-responsive elements in their promoters. Expression levels of these PgSBPs were upregulated by abiotic stresses and downregulated by ABA. Together, this study shows a comprehensive overview of $P g S B P$ s and provides vital information for elucidating the biological functions of PgSBPs.
\end{abstract}

\section{Introduction}

Plant growth is adversely affected by various abiotic stresses including drought, salinity, heat, and cold. Plants often establish physiological and metabolic defense systems to respond to these adverse conditions. In higher plants, transcription factors (TFs) play vital roles in the regulating physiological processes and adapting to environmental stresses through various signal transduction pathways (Chen and Rajewsky, 2007). SQUAMOSA promoter binding protein-like protein (SBP or SPL) genes encode a plant-specific family of TFs, which have a highly conserved SBP domain with approximately 76 amino acid residues. This conserved domain consists of two zinc-finger structures (Zn-1 and $\mathrm{Zn}-2$ ) and a nuclear localization signal (NLS) (Yamasaki et al., 2004). Two SBP genes were first identified in Antirrhinum majus with the ability for binding the promoter region of the floral meristem identity gene SQUAMOSA (Klein et al., 1996). SBP genes also play important roles in the regulation of plant architecture (Jiao et al., 2010), the vegetative phase change (Wang et al., 2009), anthocyanin biosynthesis (Gou et al., 2011), gibberellin (GA) biosynthesis and signaling (Yu et al., 2012), and stress responses (Cui et al., 2014).

MicroRNAs, non-coding 20-24 nucleotides small RNAs, induce either transcript cleavage or transcription repression (Chen, 2009). Many SBP genes are targeted by a microRNA, miR156. In Arabidopsis, this miR156/SBP module regulates root development (Cui et al., 2014) and affects secondary metabolite accumulation, which can improve stress tolerance (Yu et al., 2015). In rice, OsSPL14, which is regulated by OsmiR156, improves panicle branching, grain yield, and shoot branching (Jiao et al., 2010; Miura et al., 2010). In switchgrass, the

\footnotetext{
Abbreviations: SBP, SQUAMOSA promoter binding protein-like protein; NLS, nuclear location signal; ML, maximum-likelihood; MW, molecular weight; pI, isoelectric point; qRT-PCR, quantitative reverse transcription-PCR; ABA, abscisic acid; GA, gibberellic acid; ROS, reactive oxygen species.

* Corresponding author.

E-mail address: takano@anesc.u-tokyo.ac.jp (T. Takano).
} 
overexpression of miR156 induces aerial bud formation, while the overexpression of SPL4 suppresses bud formation and tillering (Gou et al., 2017). In alfalfa, miR156 improves drought and heat stress tolerance by silencing SPL13 (Arshad et al., 2017; Matthews et al., 2019). Another microRNA, miR529, sharing 14-16 nucleotides with miR156, was also reported to target SBPs in plants (Zhang and Ling, 2018).

Pearl millet (Pennisetum glaucum (L.) R. Br., syn. Cenchrus americanus (L.) Morrone), is a highly cross-pollinated diploid $(2 n=2 \mathrm{x}=14)$ with a draft genome size of $1.79 \mathrm{~Gb}$ (Varshney et al., 2017). It is a C4 cereal crop with nutrient-rich seeds and can grow in marginal environments characterized by low and/or unstable rainfall, poor soil conditions, and high temperature. Although the roles of SBP genes have been revealed in many plants, such as Arabidopsis (Cardon et al., 1999), rice (Xie et al., 2006), cotton (Zhang et al., 2014), foxtail millet (Bennetzen et al., 2012), pepper (Zhang et al., 2016), tobacco (Han et al., 2016) and sorghum (Chang et al., 2016), their functions in pearl millet are unclear. In order to characterize the SBP genes in pearl millet (PgSBPs), we analyzed the SBP genes in the pearl millet genome and performed phylogenetic analysis, conserved domain analysis, and the cis-element analysis for these genes. Expression patterns of PgSBPs, PgmiR156, and PgmiR529 under various conditions were also investigated. The results can help to perform further functional analysis of PgSBPs in pearl millet.

\section{Materials and methods}

\subsection{Plant materials}

One inbred pearl millet line, ICMB 843, which had been evaluated as a drought-tolerant line in the International Crop Research Institute of Semi-Arid Tropics (ICRISAT), India, was used in this study. Seeds were sown in the soil that consists of akadama (80\%), vermiculite $(10 \%)$, charcoal (8\%), slow-release (1\%), and quick-release (1\%) fertilizers. Plants were grown in a greenhouse in Tokyo, Japan, under the sunlight in July and August (with the day length of 13.5-14.5 $\mathrm{h}$ and the maximum light intensity $\sim 1300 \mu \mathrm{mol} \cdot \mathrm{m}^{-2} \cdot \mathrm{s}^{-1}$ ). The temperature and relative humidity in the greenhouse were set for $25{ }^{\circ} \mathrm{C}$ and $65 \%$, respectively. These settings kept the actual temperature $25-28{ }^{\circ} \mathrm{C}$ and the actual humidity $55-75 \%$. For gene cloning, root samples were collected from 4-week-old plants, while for expression analysis, roots and leaves were collected from 4-week-old untreated plants (Control) and plants treated with $15 \%(w / v)$ PEG6000, $250 \mathrm{mM} \mathrm{NaCl}, 42{ }^{\circ} \mathrm{C}$ and $25 \mu \mathrm{M}$ abscisic acid (ABA) for $6 \mathrm{~h}$ and $24 \mathrm{~h}$.

\subsection{Identification and bioinformatic analysis of PgSBPs}

In a previous study, the whole genome of pearl millet was sequenced, and individual gene and protein sequences were deduced from this whole genome sequence (Varshney et al., 2017). These protein sequences were submitted as the query to the HMMER hmmscan program to identify $a b$ initio the proteins with an SBP domain (Pfam accession number: PF03110). CD-search (https://www.ncbi.nlm.nih.gov/Structu re/cdd/wrpsb.cgi) and Pfam (https://pfam.xfam.org/family/PF03110) were used to confirm the presence of the SBP domain in those proteins. Sequence logos for the SBP domain were generated by WebLogo 2.8.2 (http://weblogo.berkeley.edu/logo.cgi) (Crooks et al., 2004). The molecular weight (MW) and theoretical isoelectric point (pI) of PgSBPs were calculated using the ProtParam tool on the ExPASy server (http s://web.expasy.org/protparam/). The conserved motifs were obtained by MEME 5.0.5 program (http://meme-suite.org/tools/meme) with the following parameters: a maximum number of motifs 16 ; motif width range 6-50 residues (Bailey et al., 2009). Intron/exon structures of $P g S B P$ s were determined by aligning the cDNA sequences of PgSBPs to their corresponding genomic sequences using Gene Structure Display Server 2.0 (http://gsds.cbi.pku.edu.cn/index.php) (Hu et al., 2015). The cis-acting elements in the 2000 bp promoter regions were detected by the online website PlantCARE (http://bioinformatics.psb.ugent. be/webtools/plantcare/html/) (Lescot, 2002).

\subsection{Chromosome location, the percentage identity matrix, and phylogenetic analysis}

Chromosome locations of the PgSBPs were determined by the Map Gene 2 Chromosome v2 (http://mg2c.iask.in/mg2c_v2.0/). A percentage identity matrix of PgSBP was built on the basis of the sequence alignment using Clustal Omega (https://www.ebi.ac.uk/Tools/msa/ clustalo/) and presented as a heatmap in HemI 1.0 (Deng et al., 2014). The SBP domain sequences of pearl millet, sorghum, rice, and foxtail millet were used together for the phylogenetic analysis. Multiple sequence alignment was conducted by ClustalW with default parameters in the MEGA X software. The phylogenetic tree was reconstructed by using the maximum-likelihood (ML) method with 1000 bootstrap replications based on the JTT $+\mathrm{G}$ model (Kumar et al., 2016). The resulting tree was visualized using iTOL v4 (Letunic and Bork, 2019).

\subsection{Prediction of PgSBPs targeted by PgmiRNAs}

The 290 microRNA sequences of pearl millet were obtained from previous small RNA sequencing data (Shinde et al., 2020). PgSBPs targeted by PgmiR156q or PgmiR529b were predicted by the psRNATarget server (http://plantgrn.noble.org/psRNATarget/) with default parameters (Dai et al., 2018b).

\subsection{DNA isolation and PCR amplification}

Genomic DNA was extracted from the roots of 4-week-old pearl millet plants with a DNeasy Plant Mini Kit (QIAGEN, Germany) in accordance with the manufacturer's instructions. PgSBP sequences were obtained by PCR using the genomic DNA as the template. Primers used for the PCR are listed in Table S1. The PCR products were gel-purified by FastGene Gel/PCR Extraction Kit (Genetics, Japan) and sequenced by Macrogen, Japan.

\subsection{RNA extraction and quantitative reverse transcription-PCR ( $q R T$ - PCR) analysis}

Total RNA was extracted from leaves and roots of the 4-week-old pearl millet plants with a NucleoSpin RNA Plant kit (MACHEREYNAGEL, Germany) according to the manufacturer's instructions. cDNA was synthesized from $1 \mu \mathrm{g}$ of the total RNA with Prime Script Reverse Transcriptase (Takara, Japan) and the oligo (dT) primer. The expression levels of PgSBPs were quantified by the quantitative reverse transcription-PCR (qRT-PCR), which was performed with the StepOne Real-Time PCR System (Applied Biosystems, USA) and the TB Green Premix Ex Taq ${ }^{\mathrm{TM}}$ (Takara, Japan). The PCR cycle was: $95^{\circ} \mathrm{C}$ for $30 \mathrm{~s}$, followed by 40 cycles at $95^{\circ} \mathrm{C}$ for $5 \mathrm{~s}$ and $60^{\circ} \mathrm{C}$ for $30 \mathrm{~s}$, and then a dissociation curve analysis was performed at $95^{\circ} \mathrm{C}$ for $60 \mathrm{~s}, 55^{\circ} \mathrm{C}$ for 30 $\mathrm{s}$, and $95^{\circ} \mathrm{C}$ for $30 \mathrm{~s}$. The Ubiquitin (UBQ) gene (GenBank accession number XM_004977046.2) (Anup et al., 2017) was used as the reference gene. Gene-specific primers for the PgSBPs were designed by the PrimerBLAST online server (https://www.ncbi.nlm.nih.gov/tools/primer-bl ast/) based on the MIQE guidelines (http://rdml.org/miqe). The primers are listed in Supplementary Table S2. The comparative Ct method was used to quantify expression levels (Schmittgen and Livak, 2008). For microRNA expression analysis, the cDNA was reversetranscribed from $2 \mu \mathrm{g}$ of the total RNA with the Mir-XTM miRNA First-Strand Synthesis Kit (Takara, Japan). For the qRT-PCR, the entire sequences of mature microRNAs were used for forward primers, and the mRQ3' primer from the kit was used as the reverse primer. U6 snRNA from the kit was used as the reference RNA. The PCR was conducted as described above. 
Table 1

The 18 PgSBPs identified in pearl millet and their sequence characteristics.

\begin{tabular}{lllllll}
\hline Name & Gene ID & $\begin{array}{l}\text { Gene } \\
\text { length } \\
\text { (bp) }\end{array}$ & $\begin{array}{l}\text { CDS } \\
\text { length } \\
\text { (bp) }\end{array}$ & $\begin{array}{l}\text { No. of } \\
\text { amino } \\
\text { acids (aa) }\end{array}$ & MW & pI \\
\hline PgSBP1 & MW561430 & 12,237 & 2568 & 855 & 94.01 & 5.59 \\
PgSBP2 & MW561431 & 2966 & 1314 & 437 & 45.84 & 6.75 \\
PgSBP3 & MW561432 & 2994 & 858 & 285 & 31.10 & 9.72 \\
PgSBP4 & MW561433 & 3467 & 1482 & 493 & 52.46 & 9.14 \\
PgSBP5 & MW561434 & 2546 & 1164 & 387 & 39.23 & 9.58 \\
PgSBP6 & MW561435 & 3153 & 1191 & 396 & 43.16 & 7.47 \\
PgSBP7 & MW561436 & 4390 & 858 & 285 & 30.30 & 8.33 \\
PgSBP8 & MW561437 & 2463 & 1296 & 431 & 45.84 & 9.24 \\
PgSBP9 & MW561438 & 3027 & 1302 & 433 & 47.42 & 8.95 \\
PgSBP10 & MW561439 & 2082 & 846 & 281 & 29.98 & 9.34 \\
PgSBP11 & MW561440 & 4262 & 2424 & 807 & 90.07 & 6.78 \\
PgSBP12 & MW561441 & 2995 & 540 & 179 & 19.03 & 10.19 \\
PgSBP13 & MW561442 & 2947 & 501 & 166 & 17.41 & 9.98 \\
PgSBP14 & MW561443 & 3625 & 774 & 257 & 27.73 & 10.28 \\
PgSBP15 & MW561444 & 4231 & 2964 & 987 & 10.89 & 5.96 \\
PgSBP16 & MW561445 & 5312 & 2934 & 977 & 10.67 & 5.54 \\
PgSBP17 & MW561446 & 2670 & 864 & 287 & 30.41 & 8.95 \\
PgSBP18 & MW561447 & 3245 & 966 & 322 & 34.01 & 6.56 \\
\hline & & & & & & \\
\hline
\end{tabular}

\section{Results}

3.1. Identification, molecular cloning, and gene feature analysis of PgSBPs

Eighteen SBP genes (PgSBP1-18) were identified in the pearl millet genome and their sequences were deposited in the NCBI database (Table 1). The lengths of these genomic sequences varied from $2082 \mathrm{bp}$ (PgSBP10) to 12,237 bp (PgSBP1). The coding sequences of PgSBPs ranged from $501 \mathrm{bp}(P g S B P 13)$ to $2964 \mathrm{bp}$ (PgSBP15), with the deduced proteins ranging from 166 to 987 amino acids in length and from 10.67 (PgSBP16) to 94.01 (PgSBP1) $\mathrm{kDa}$ in protein mass. The predicted pI of the PgSBPs varied from 5.54 (PgSBP16) to 10.28 (PgSBP14). Among all the PgSBPs, 12 members are basic proteins with pI values more than 7.0 and the rest are acidic proteins (Table 1). PgSBPs in general have low identities to each other, except that PgSBP7 and PgSBP13 share 66\% identity, PgSBP7 and PgSBP14 share 62\% identity, and PgSBP13 and
PgSBP17 share $62 \%$ identity (Fig. S1 and Table S3). Fourteen PgSBPs (PgSBP1-14) were unevenly distributed on 7 chromosomes with one gene on Chr1 and Chr4; two on Chr3, Chr5, and Chr6; and three on Chr2 and Chr7 (Fig. 1). PgSBP15, PgSBP16, PgSBP17, and PgSBP18 were located on scaffolds (i.e., genomic sequences that have not been assigned to any chromosome) 2013, 2474, 2484, and 4011, respectively.

\subsection{Sequence alignments and phylogenetic analysis of SBP domains}

SBP domains have high similarity with each other and most of the PgSBPs have both $\mathrm{Zn}-1$ and $\mathrm{Zn}-2$ as well as an NLS in their SBP domains (Fig. 2). However, PgSBP7, PgSBP10, PgSBP14, and PgSBP18 lack Zn-1 and a part of Zn-2 (Fig. 2A). PgSBP11, PgSBP15, and PgSBP16 contain not only a SBP domain but also an ankyrin (ANK) domain (Fig. S2), which is associated with protein-protein interactions (Michaely and Bennett, 1992).

A phylogenetic tree was constructed using the highly conserved SBPdomains of the 18 PgSBPs, 27 Setaria italica SBP proteins (SiSBPs), 39 Sorghum bicolor SBP proteins (SbSBPs), and 19 Oryza sativa SBP proteins (OsSBPs). The resulting tree suggests that the 18 PgSBPs can be classified into seven groups (group I to VII, Fig. 3) and that all of these groups contain at least one SBP member from each species. Group VII is the largest group with six PgSBPs included. Groups I and II are the smallest groups with only one PgSBP member included.

\subsection{Structural organization and conserved motif analysis of PgSBPS}

Intron/exon structures of the $18 \mathrm{PgSBPs}$ were analyzed by their genomic sequences and protein-coding sequences. The number of exons among PgSBPs ranged from 2 (PgSBP12 and PgSBP13) to 11 (PgSBP16). The PgSBPs in the same group in the phylogenetic tree shared a similar intron/exon structure (Fig. 4).

In addition to the SBP and ANK domains, 16 motifs were identified for the 18 PgSBPs (Fig. 5, Table S5). The number of these motifs in each PgSBPs varies from 1 (PgSBP3) to 10 (PgSBP11 and PgSBP16) and the proteins in group IV possess the largest numbers of conserved motifs. Motif 1 , motif 2, and motif 3 represent the $\mathrm{N}$ terminal, $\mathrm{C}$ terminal, and middle parts of the SBP domain, respectively. Some of these motifs are

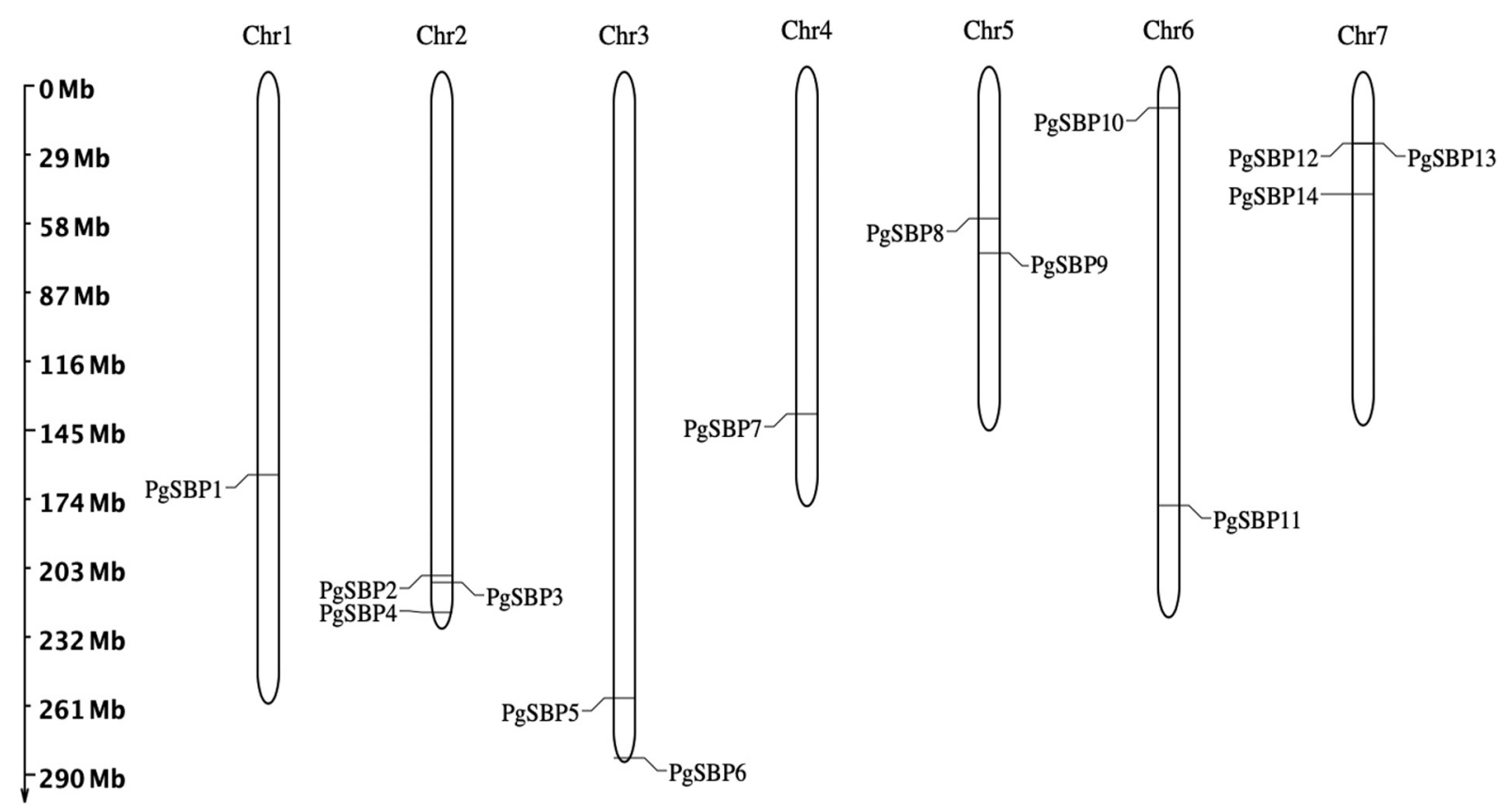

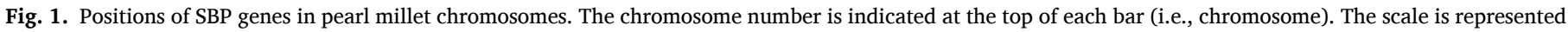
in mega base $(\mathrm{Mb})$. 


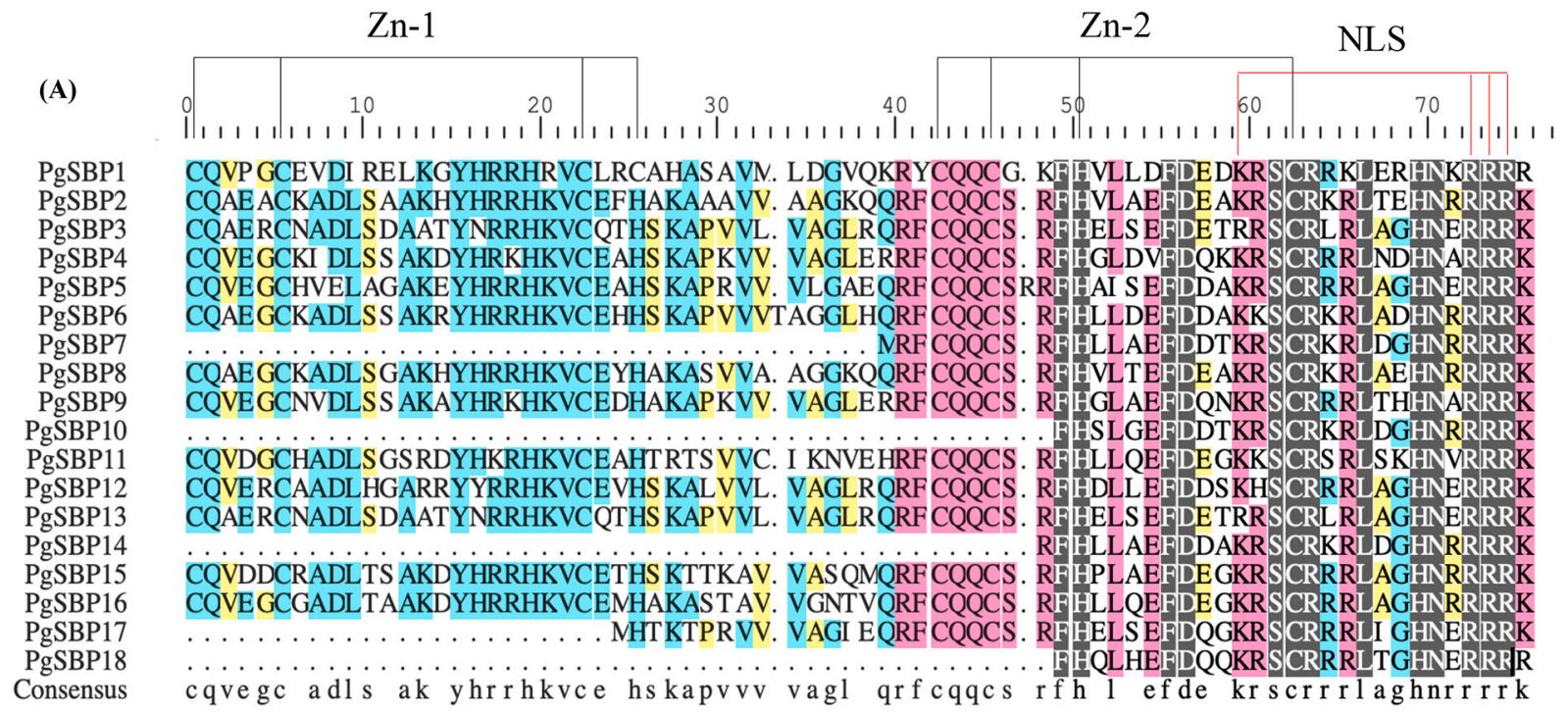

(B)

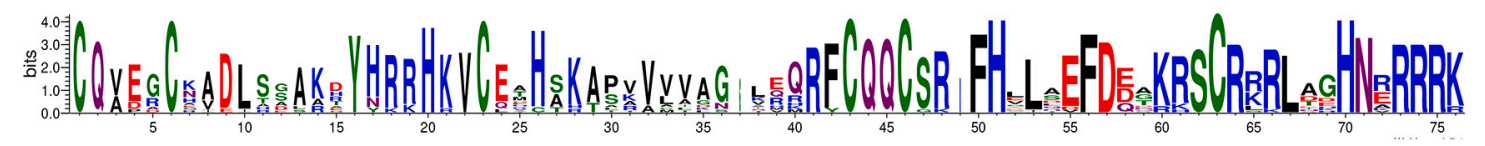

Fig. 2. Alignment of PgSBPs. (A) Multiple alignment of SBP domains of PgSBPs obtained with DNAMAN software. The two conserved zinc-finger structures (Zn-1 and Zn-2) and NLSs are indicated. (B) Sequence logos for the SBP domain of PgSBPs. The overall height of each stack represents the extent of conservation at each position, while the height of the letters within each stack indicates the relative frequency of the corresponding amino acid.

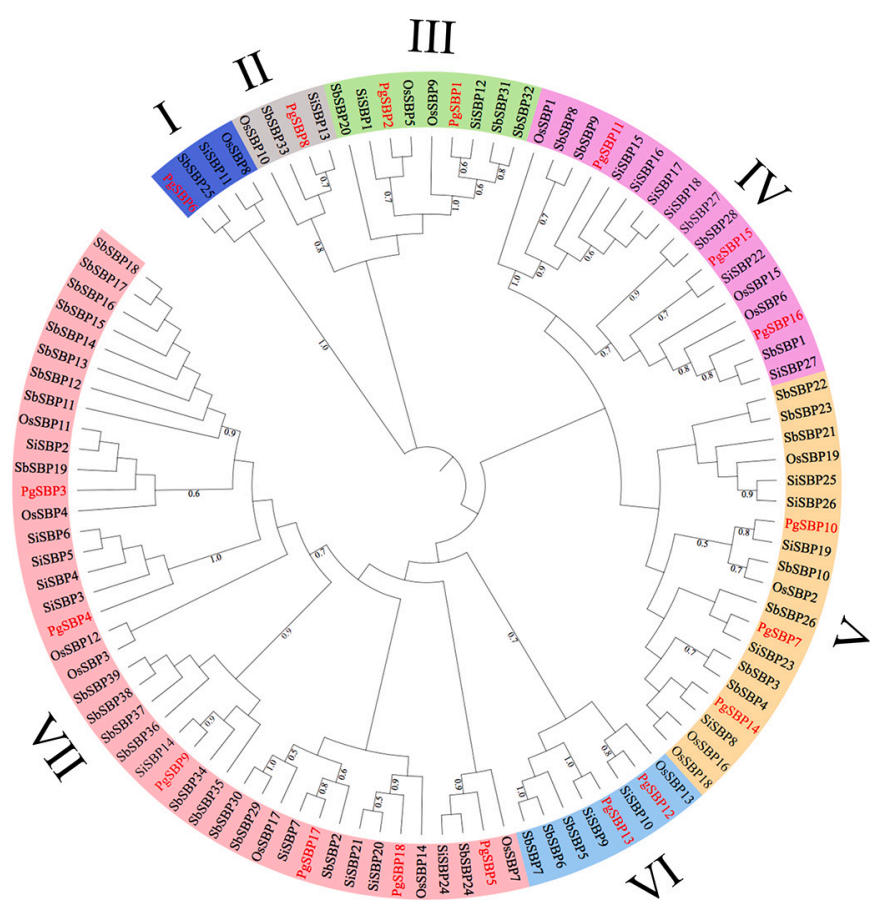

Fig. 3. The maximum likelihood (ML) phylogenetic tree of SBP proteins from pearl millet (PgSBP), sorghum (SbSBP), foxtail millet (SiSBP) and rice (OsSBP). The SBP domain sequences of all genes used for phylogenetic tree construction are listed in Supplementary Table S4.

specific to some groups. These data support the idea that the PgSBPs in the same group have similar functions.

\subsection{Cis-elements analysis in the promoter regions of PgSBPs}

The cis-elements in the 2000-bp promoter regions of PgSBPs were identified (Table S6) and were categorized into six groups based on the predicted functions shown in Fig. 6. The cis-elements identified include the elements regulating hormone responsiveness, such as the ABAresponsive elements (ABREs), the methyl jasmonate (MeJA)-responsive elements (CGTCA-motifs), auxin-responsive elements (TGA-elements and AuxRR-core elements), salicylic acid (SA)-responsive elements (TCA-element and SARE) and GA-responsive elements (GARE motifs, P-boxes, and TATC-boxes) (Table S6). Stress-associated cis-elements were also identified, such as the low-temperature responsive elements (LTREs), the drought-responsive elements (DREs), the MYB binding sites (MBSs), and TC-rich repeats. All the PgSBPs except PgSBP3 and $P g S B P 18$ have some of those cis-elements, raising the possibility that most of the PgSBPs are either induced or repressed by phytohormones and/or abiotic stresses.

\subsection{The regulation of the PgSBPs transcript by PgmiR156q and PgmiR529b}

Nine out of the 18 PgSBPs (PgSBP3, PgSBP4, PgSBP5, PgSBP7, $P g S B P 9, P g S B P 10, P g S B P 14, P g S B P 17$, and PgSBP18), which encode PgSBPs in the groups V and VII, were found to be potential targets of a pearl millet miR156, PgmiR156q (Fig. 7A). All these genes, except PgSBP4, are also potential targets of a pearl millet miR529, PgmiR529b (Fig. S3). The distances between their SBP domain-coding sequences and putative microRNA binding sites were similar between these PgSBPs (326 bp-418 bp) (Fig. 7B). These results raise the possibility that these microRNA target sites originated from the common ancestor.

\subsection{Expression profiles of PgSBPs, PgmiR156q, and PgmiR529b in different tissues under various abiotic stresses}

In order to explore the possible functions of PgSBPs, the expression profiles of all PgSBPs were investigated in root and leaf tissues under salinity-stressed, drought-stressed, heat-stressed, and ABAsupplemented conditions with qRT-PCR (Fig. 8). In leaf tissues, two genes, PgSBP8 and PgSBP10, were upregulated by salinity stress and $P g S B P 2$ was upregulated by drought stress. Twelve out of 18 PgSBPs 


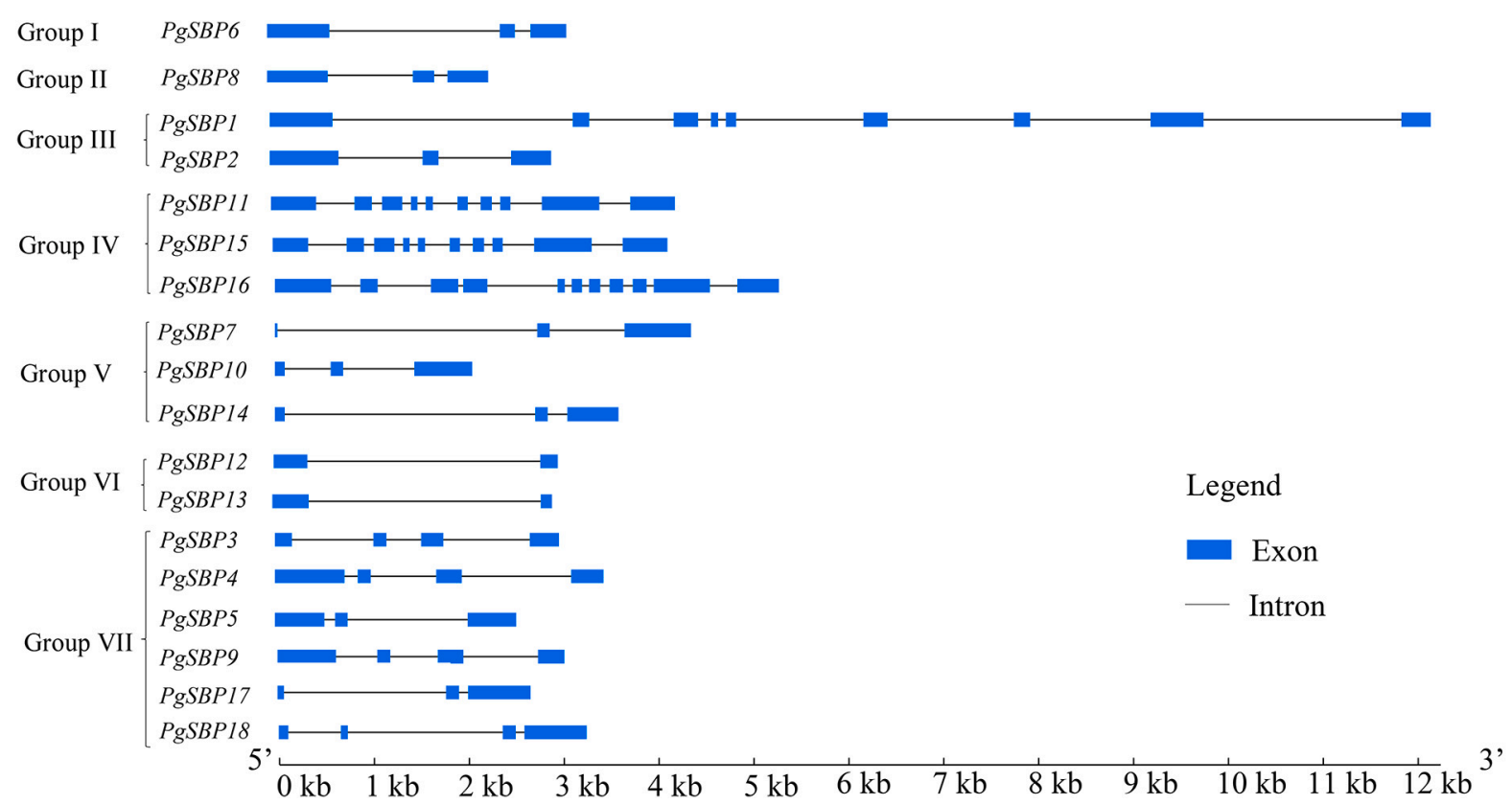

Fig. 4. Exon/intron structures of PgSBPs. Exons and introns are indicated by blue rectangles and black horizontal lines, respectively. (For interpretation of the references to colour in this figure legend, the reader is referred to the web version of this article.)

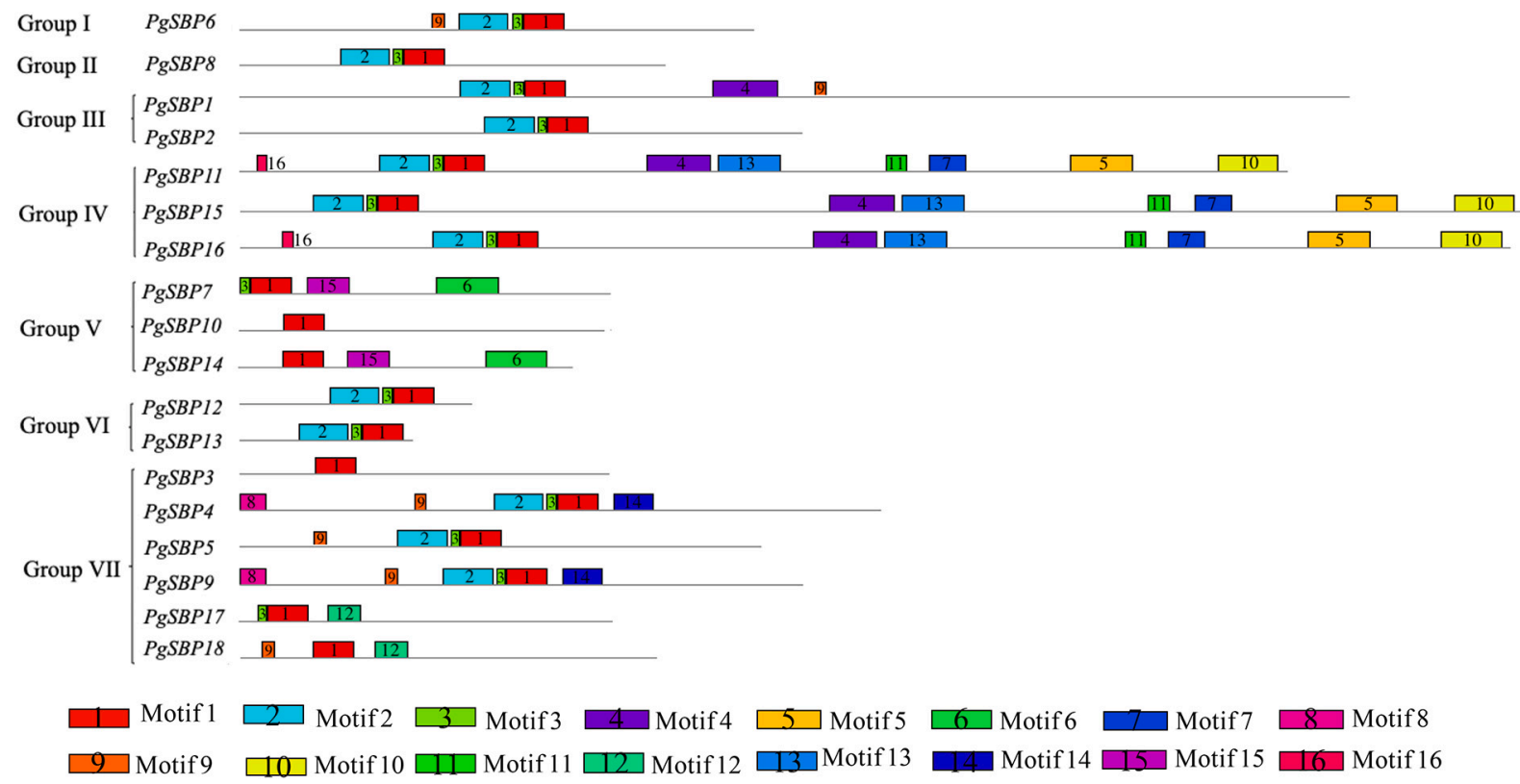

Fig. 5. Distribution of conversed motifs in PgSBPs. Motifs indicated by boxes were detected by MEME and the number in boxes (1 to 16) represents motif 1 to motif 16, respectively. Box sizes indicate the length of the motifs and the consensus sequences of these motifs are presented in Table S5.

were upregulated by heat stress. In roots, most of the PgSBPs were upregulated by salinity, drought, and heat stresses. According to previous RNA sequencing data (Dudhate et al., 2018), the absolute expression levels of PgSBP3, PgSBP10, PgSBP11, PgSBP15, and PgSBP16 were higher than the other $P g S B P$ s in root tissues under a control condition (Fig. S4). All these 5 PgSBPs were significantly induced by salinity, drought, and heat stresses (Fig. 8). The expression of PgSBP2 in roots was 205 and 119 times stronger in the presence of salinity and drought stresses, respectively, compared to expression in control roots. The expression of PgSBP7 and PgSBP13 in roots was also 109 times stronger in the presence of salinity stress than in control roots (Fig. 8). Thus, although the absolute expression levels of these genes in roots are low (Fig. S4), they may regulate salinity and/or drought stress responsiveness. Further studies are necessary to clarify their functions.

Expression of pearl millet miR156 and miR529 (PgmiR156q and PgmiR529b) was also examined under the same conditions. In general, the expression patterns of PgmiR156q and PgmiR529b were similar to each other and opposite to PgSBPs (Fig. 8). For instance, PgmiR156q and PgmiR529b were downregulated in roots by the salinity and drought stresses, which upregulated the PgmiR156q- and PgmiR529b- targeted $P g S B P$ s. These results support the idea that the expression levels of some of the PgSBPs are regulated by PgmiR156q and PgmiR529b. 


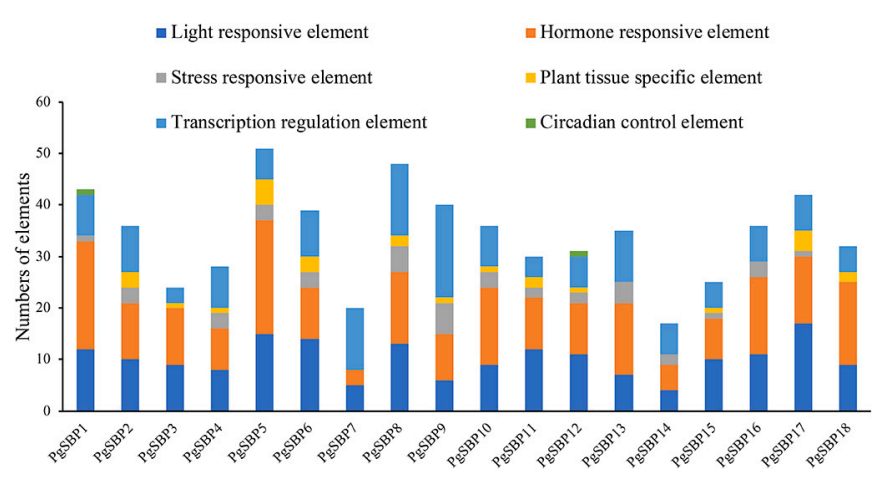

Fig. 6. Cis-elements in promoter regions of PgSBPs. The elements were identified from $2000 \mathrm{bp}$ upstream promoter regions and the detailed information of these cis-elements is given in Table S6.

\subsection{Expression profiles of PgSBPs, PgmiR156q, and PgmiR529b in different tissues under ABA treatment}

Expression levels of the 18 PgSBPs were analyzed in roots and leaves treated with $25 \mu \mathrm{M} \mathrm{ABA}$ (Fig. 9). All of these PgSBPs were downregulated by a 24-h ABA treatment in leaf tissues and many of these genes were also downregulated by the same treatment in root tissues. In contrast, the expression levels of PgmiR156q and PgmiR529b were increased in both leaves and roots by the ABA treatment. These results suggest that ABA induces PgmiR156q and PgmiR529b-PgSBPs and thereby downregulates $P g S B P$ s.

\section{Discussion}

In this study, $18 \mathrm{PgSBPs}$ were identified in the pearl millet genome (Table 1). This number is similar to the numbers of SBP-like genes in Arabidopsis thaliana (16) (Cardon et al., 1999), Betula luminifera (18) (Lin et al., 2018), Oryza sativa (19) (Xie et al., 2006), Camellia sinensis (20) (Wang et al., 2018a), and Carica papaya (14) (Xu et al., 2020). However, the number is smaller than the numbers of SBP-like genes in Setaria italica (27) (Tian et al., 2017), Zea mays (31) (Mao et al., 2016), and Glycine max (41) (Tripathi et al., 2017). This may be because the SBPs evolved in a species-specific manner.

The 18 PgSBPs were divided into seven groups based on phylogenetic analysis (Fig. 3). This is consistent with the classification of SBPs of other species, such as cotton (Zhang et al., 2014), rice (Xie et al., 2006) and maize (Mao et al., 2016). PgSBPs in group IV have the ANK domain, which is predicted to promote a protein-protein interaction (Fig. S2). The ANK domain is also present in 6 SBPs in maize, 3 SBPs in luminifera, and 6 SBPs in cotton (Mao et al., 2016; Lin et al., 2018; Li and Lu, 2014). These findings suggest that the ANK domain is relevant to the functions of these SBPs.

Many of the 19 rice SBPS (OSSPL1-19, which correspond to OsSBP1-19, respectively, in Fig. 3) have been characterized. OsSPL3 can be downregulated by miR156 and OSSPL3 induces the expression of the WRKY71 transcription factor gene, thereby negatively regulating cold stress tolerance (Zhou and Tang, 2019). OSSPL3 and OSSPL12 are also known as positive regulators of crown root development (Shao et al., 2019). OsSPL6 represses the expression of an endoplasmic reticulum stress sensor gene, OsIRE1, thereby regulating panicle development (Wang et al., 2018b). OSSPL7 can also be downregulated by miR156 and OSSPL7 directly regulates the expression of the auxin-related gene OsGH3.8, thereby decreasing the tiller number (Dai et al., 2018a). OsSPL8 regulates the development of ligules and auricles in leaves (Lee et al., 2007). OsSPL9 is a positive regulator of copper transporter genes and can increase copper contents in shoots (Tang et al., 2016). OsSPL10 induces trichome initiation in leaves and glumes and decreases salt tolerance (Lan et al., 2019). OsSPL13 can also be downregulated by miR156 and increases cell sizes in the grain hull, thereby increasing grain length and yield ( $\mathrm{Si}$ et al., 2016). OsSPL14 decreases the tiller number and increases stem strength, lodging resistance, grain number, and grain weight (Jiao et al., 2010; Miura et al., 2010). OsSPL16

\section{(A)}
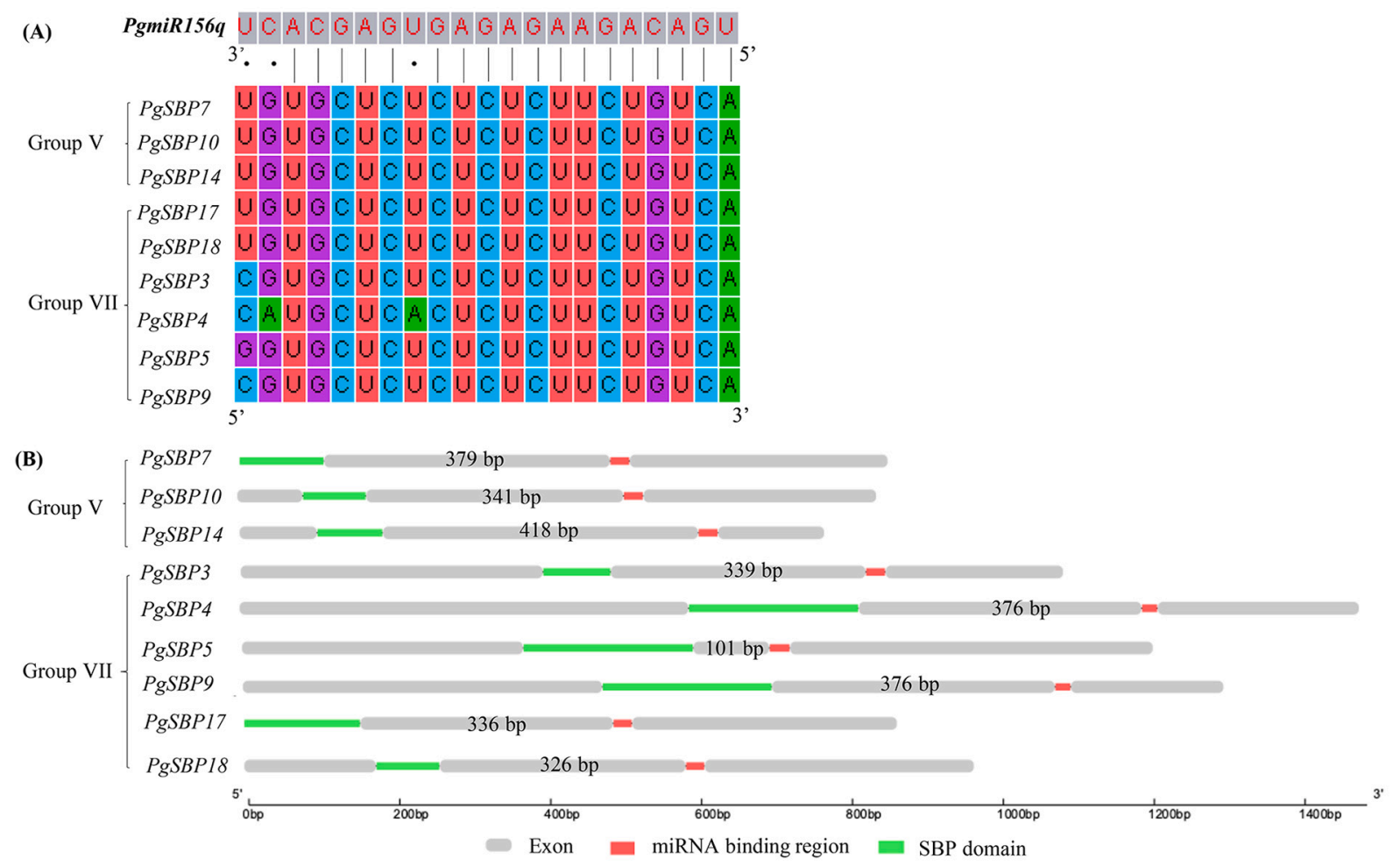

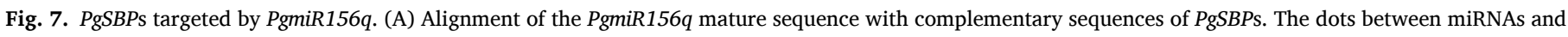
targeted PgSBP sequences indicate mismatches. (B) The positions of miRNA binding regions and SBP domains in the sequences of PgSBPs. 

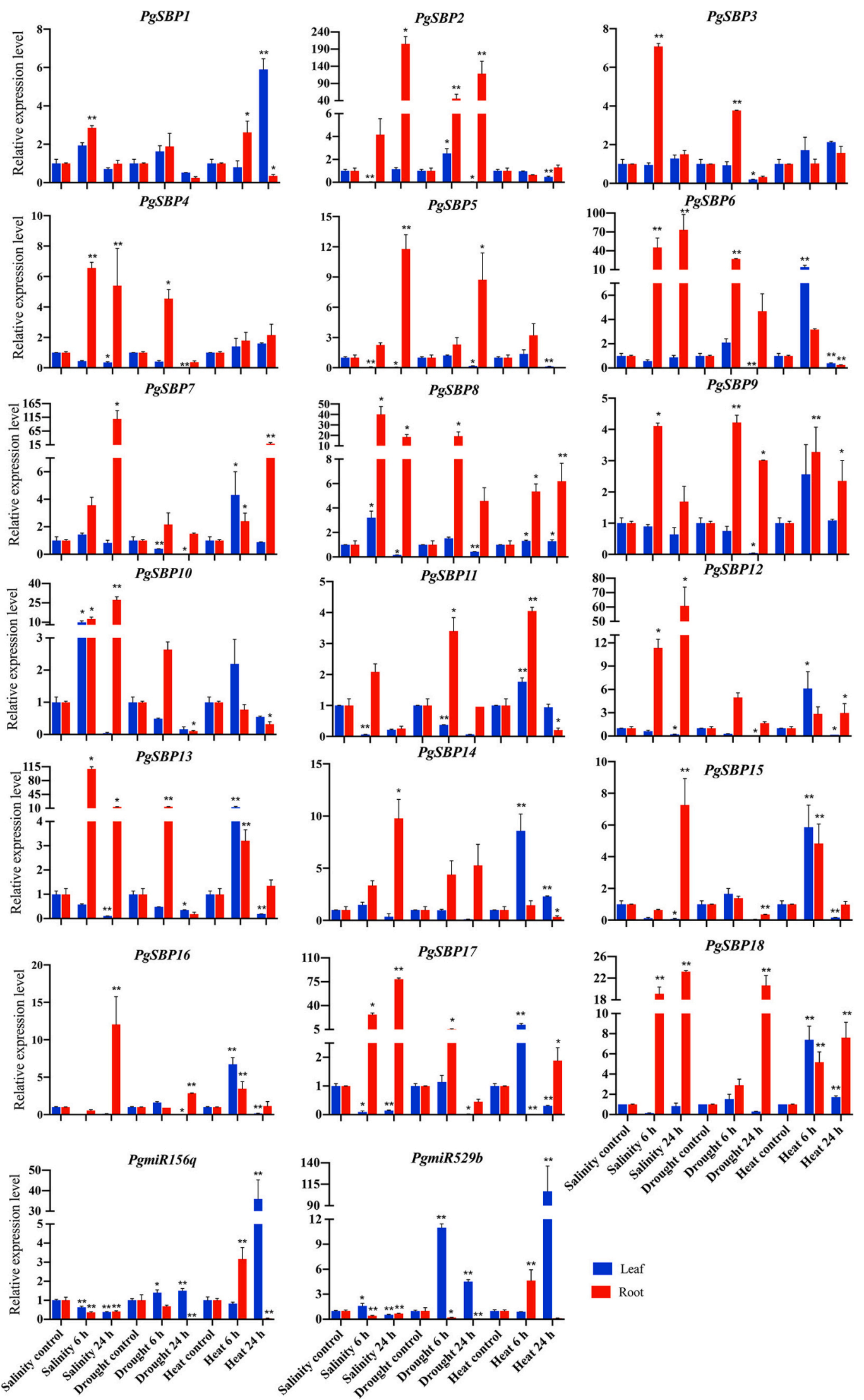

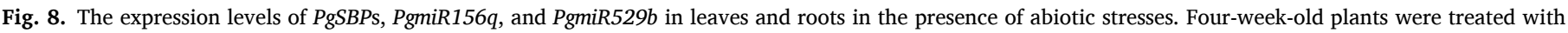

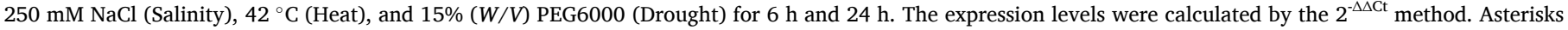
indicate significant differences from each control. ${ }^{*} P<0.05,{ }^{*} P<0.01$, Student's $t$-test. 

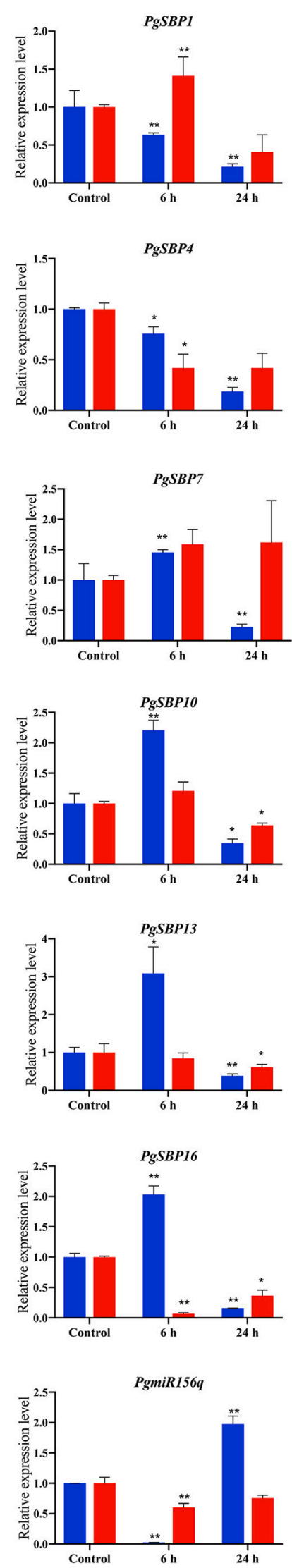
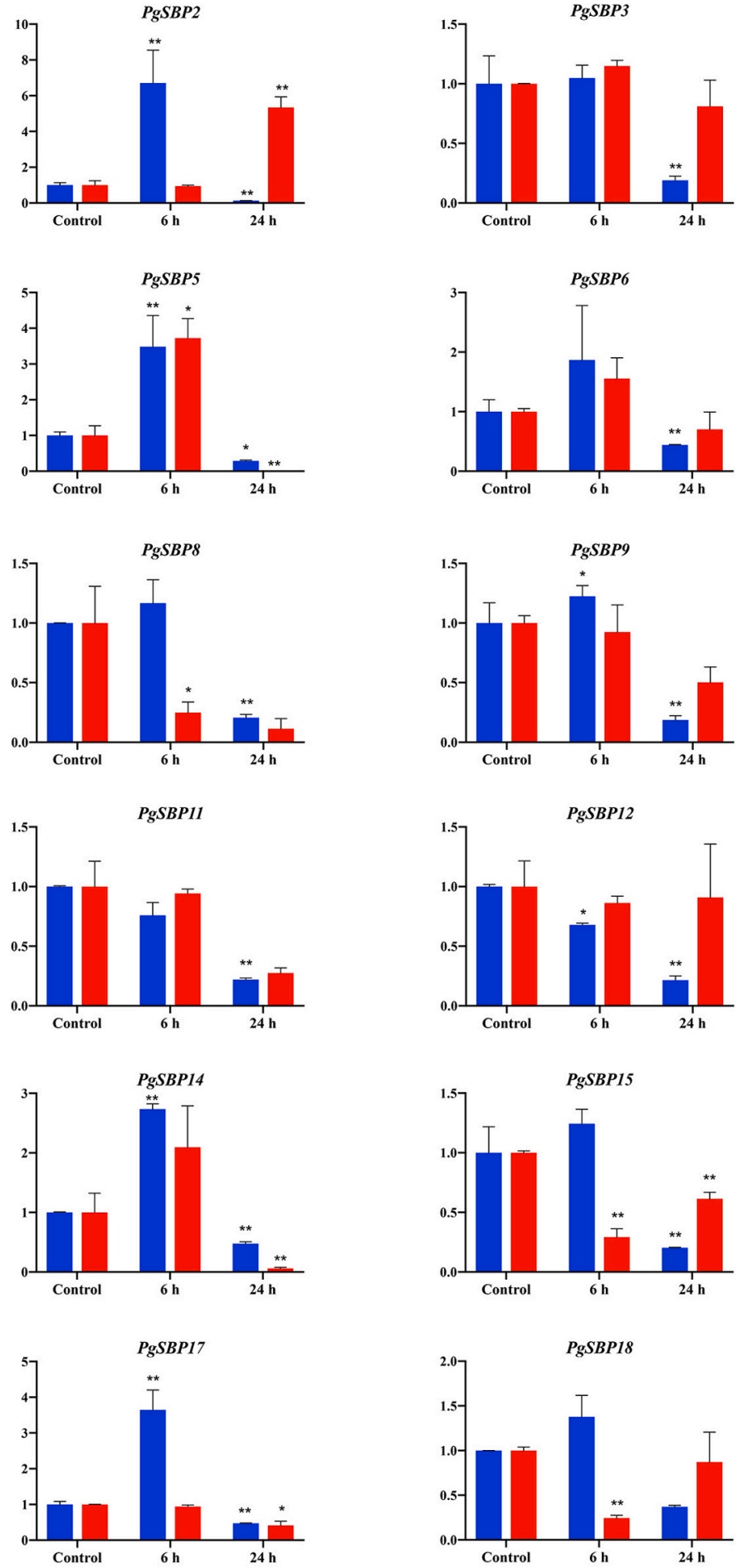

Fig. 9. The expression level of PgSBPs, PgmiR156q, and PgmiR529b in leaves and roots in the presence of ABA. Four-week-old plants were treated with $25 \mu \mathrm{M}$ ABA for

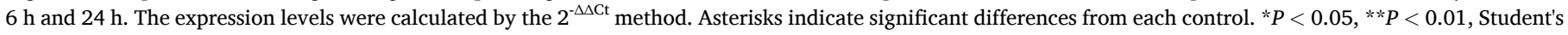
$t$-test. 
promotes cell proliferation in grains, thereby increasing grain width and yield (Wang et al., 2012). OsSPL14 and OsSPL16 as well as their close homolog OSSPL2 are downregulated by miR156 (Xie et al., 2006) and miR529 (Yue et al., 2017). OSSPL18 can also be downregulated by miR156, and OsSPL18 increases panicle length, grain thickness, the grain number yet decreases the tiller number (Yuan et al., 2019). Our data suggest that pearl millet has close homologs of all of these rice SBP genes (Fig. 3). Physiological functions of SBP are likely to be conserved in pearl millet and rice, but the functions of the pearl millet SBPs need to be characterized in detail in the future.

Some SBP genes in other species play roles in regulating plant responses to abiotic stresses and phytohormones. For instance, overexpression of an SBP gene (VPSBP16) from a Chinese wild Vitis species in Arabidopsis enhances the tolerance to salinity and drought stresses (Hou et al., 2018). Several SBPs in Betula luminifera interact with two DELLA proteins and regulate GA responses (Lin et al., 2018). The overexpression of a B. luminifera SBP, BpSPL9, enhances tolerance to drought and salinity by scavenging reactive oxygen species (ROS) in B. platyphylla Suk (Ning et al., 2017). Some of the 14 SBP genes in papaya (Carica papaya) are induced at a specific stage in fruit ripening and this process can be controlled by the phytohormone ethylene (Xu et al., 2020). TaSPL14, a close OsSPL14 homolog in wheat (Triticum aestivum), does not regulate the tiller number but binds to the promoters of the ethylene-related genes TaEIL1, TaRAP2.11, and TaERF1 and increases their expression, thereby regulating plant height, panicle length, the spikelet number, and grain weight (Cao et al., 2021). In this study, ABA-responsive elements and stress-responsive elements were found in most of the $P g S B P$ promoters and ABA treatments reduced the expression levels of these genes. In addition, the expression levels of most $P g S B P s$ in roots were increased by various abiotic stresses. Therefore, we hypothesize that PgSBPs play essential roles in pearl millet responses to abiotic stresses and phytohormones.

Supplementary data to this article can be found online at https://doi. org/10.1016/j.plgene.2021.100313.

\section{Funding}

This work was supported by JSPS KAKENHI Grant Number JP19H02928.

\section{Declaration of Competing Interest}

The authors declare no conflict of interest.

\section{Acknowledgements}

We are grateful to ICRISAT, India for providing the pearl millet seeds for this study. Pei Yu also thanks financial support by the China Scholarship Council (CSC).

\section{References}

Anup, P.C., Melvin, P., Kini, K.R., 2017. Reference gene selection and validation for gene expression studies in downy mildew infected pearl millet by quantitative real-time PCR. Australas. Plant Pathol. 46, 441-452. https://doi.org/10.1007/s13313-0170506-z.

Arshad, M., Feyissa, B.A., Amyot, L., Aung, B., Hannoufa, A., 2017. MicroRNA156 improves drought stress tolerance in alfalfa (Medicago sativa) by silencing SPL13. Plant Sci. 258, 122-136. https://doi.org/10.1016/j.plantsci.2017.01.018.

Bailey, T.L., Boden, M., Buske, F.A., Frith, M., Grant, C.E., Clementi, L., Ren, J., Li, W.W., Noble, W.S., 2009. MEME suite: tools for motif discovery and searching. Nucleic Acids Res. 37, 202-208. https://doi.org/10.1093/nar/gkp335.

Bennetzen, J.L., Schmutz, J., Wang, H., Percifield, R., Hawkins, J., Pontaroli, A.C., Estep, M., Feng, L., Vaughn, J.N., Grimwood, J., Jenkins, J., Barry, K., Lindquist, E., Hellsten, U., Deshpande, S., Wang, X., Wu, X., Mitros, T., Triplett, J., Yang, X., Ye, C.Y., Mauro-Herrera, M., Wang, L., Li, P., Sharma, M., Sharma, R., Ronald, P.C., Panaud, O., Kellogg, E.A., Brutnell, T.P., Doust, A.N., Tuskan, G.A., Rokhsar, D. Devos, K.M., 2012. Reference genome sequence of the model plant Setaria. Nat. Biotechnol. 30, 555-561. https://doi.org/10.1038/nbt.2196.
Chang, J., Yan, F., Qiao, L., Zheng, J., Zhang, F., Liu, Q., 2016. Genome-wide identification and expression analysis of SBP-box gene family in Sorghum bicolor L. Yi chuan = Hered 38, 569-580. https://doi.org/10.16288/j.yczz.16-008.

Cao, J., Liu, K., Song, W., Zhang, J., Yao, Y., Xin, M., Hu, Z., Peng, H., Ni, Z., Sun, Q., Du, J., 2021. Pleiotropic function of the SQUAMOSA PROMOTER-BINDING PROTEIN-LIKE gene TaSPL14 in wheat plant architecture. Planta 253, 1-12. https:// doi.org/10.1007/s00425-020-03531-x.

Cardon, G., Höhmann, S., Klein, J., Nettesheim, K., Saedler, H., Huijser, P., 1999. Molecular characterisation of the Arabidopsis SBP-box genes. Gene 237, 91-104. https://doi.org/10.1016/S0378-1119(99)00308-X.

Chen, K., Rajewsky, N., 2007. The evolution of gene regulation by transcription factors and microRNAs. Nat. Rev. Genet. 8, 93-103. https://doi.org/10.1038/nrg1990.

Chen, X., 2009. Small RNAs and their roles in plant development. Annu. Rev. Cell Dev. Biol. 25, 21-44. https://doi.org/10.1146/annurev.cellbio.042308.113417.

Crooks, G., Hon, G., Chandonia, J., Brenner, S., 2004. NCBI GenBank FTP Site $\backslash$ nWebLogo: a sequence logo generator. Genome Res. 14, 1188-1190. https://doi. org/10.1101/gr.849004.1.

Cui, L.G., Shan, J.X., Shi, M., Gao, J.P., Lin, H.X., 2014. The miR156-SPL9-DFR pathway coordinates the relationship between development and abiotic stress tolerance in plants. Plant J. 80, 1108-1117. https://doi.org/10.1111/tpj.12712.

Dai, X., Zhuang, Z., Zhao, P.X., 2018b. PsRNATarget: a plant small RNA target analysis server (2017 release). Nucleic Acids Res. 46, W49-W54. https://doi.org/10.1093/ nar/gky316.

Dai, Z., Wang, J., Yang, X., Lu, H., Miao, X., Shi, Z., 2018a. Modulation of plant architecture by the miR156f-OsSPL7-OsGH3.8 pathway in rice. J. Exp. Bot. 69, 5117-5130. https://doi.org/10.1093/jxb/ery273.

Deng, W., Wang, Y., Liu, Z., Cheng, H., Xue, Y., 2014. HemI: a toolkit for illustrating heatmaps. PLoS One 9, e1119888. https://doi.org/10.1371/journal.pone.0111988.

Dudhate, A., Shinde, H., Tsugama, D., Liu, S., Takano, T., 2018. Transcriptomic analysis reveals the differentially expressed genes and pathways involved in drought tolerance in pearl millet [pennisetum glaucum (1.) r. Br]. PLoS One 13, e0195908. https://doi.org/10.1371/journal.pone.0195908.

Gou, J., Fu, C., Liu, S., Tang, C., Debnath, S., Flanagan, A., Ge, Y., Tang, Y., Jiang, Q., Larson, P.R., Wen, J., Wang, Z.Y., 2017. The miR156-SPL4 module predominantly regulates aerial axillary bud formation and controls shoot architecture. New Phytol. 829-840. https://doi.org/10.1111/nph.14758.

Gou, J.Y., Felippes, F.F., Liu, C.J., Weigel, D., Wang, J.W., 2011. Negative regulation of anthocyanin biosynthesis in Arabidopsis by a miR156-targeted SPL transcription factor. Plant Cell 23, 1512-1522. https://doi.org/10.1105/tpc.111.084525.

Han, Y.Y., Ma, Y.Q., Li, D.Z., Yao, J.W., Xu, Z.Q., 2016. Characterization and phylogenetic analysis of fifteen NtabSPL genes in Nicotiana tabacum L. cv. Qinyan95. Dev. Genes Evol. 226, 1-14. https://doi.org/10.1007/s00427-015-0522-3.

Hou, H., Jia, H., Yan, Q., Wang, X., 2018. Overexpression of a SBP-box gene (VPSBP16) from chinese wild vitis species in Arabidopsis improves salinity and drought stress tolerance. Int. J. Mol. Sci. 19, 940. https://doi.org/10.3390/ijms19040940.

Hu, B., Jin, J., Guo, A.Y., Zhang, H., Luo, J., Gao, G., 2015. GSDS 2.0: an upgraded gene feature visualization server. Bioinformatics 31, 1296-1297. https://doi.org/ 10.1093/bioinformatics/btu817.

Jiao, Y., Wang, Y., Xue, D., Wang, J., Yan, M., Liu, G., Dong, G., Zeng, D., Lu, Z., Zhu, X., Qian, Q., Li, J., 2010. Regulation of OsSPL14 by OsmiR156 defines ideal plant architecture in rice. Nat. Genet. 42, 541-544. https://doi.org/10.1038/ng.591.

Klein, J., Saedler, H., Huijser, P., 1996. A new family of DNA binding proteins includes putative transcriptional regulators of the Antirrhinum majus floral meristem identity gene SQUAMOSA. MGG Mol. Gen. Genet. 250, 7-16. https://doi.org/10.1007/ s004380050046.

Kumar, S., Stecher, G., Tamura, K., 2016. MEGA7: molecular evolutionary genetics analysisversion 7.0 for bigger datasets. Mol. Biol. Evol. 33, 1870-1874. https://doi. org/10.1093/molbev/msw054.

Lan, T., Zheng, Y., Su, Z., Yu, S., Song, H., Zheng, X., Lin, G., Wu, W., 2019. OsSPL10, a SBP-box gene, plays a dual role in salt tolerance and trichome formation in rice (Oryza sativa L.). G3 Genes Genomes Genet. 9, 4107-4114. https://doi.org/10.1534/ g3.119.400700.

Lee, J., Park, J.J., Kim, S.L., Yim, J., An, G., 2007. Mutations in the rice liguleless gene result in a complete loss of the auricle, ligule, and laminar joint. Plant Mol. Biol. 65, 487-499. https://doi.org/10.1007/s11103-007-9196-1.

Lescot, M., 2002. PlantCARE, a database of plant cis-acting regulatory elements and a portal to tools for in silico analysis of promoter sequences. Nucleic Acids Res. 30, 325-327. https://doi.org/10.1093/nar/30.1.325.

Letunic, I., Bork, P., 2019. Interactive tree of life (iTOL) v4: recent updates and new developments. Nucleic Acids Res. 47, W256-W259. https://doi.org/10.1093/nar/ gkz239.

Li, C., Lu, S., 2014. Molecular characterization of the SPL gene family in Populus trichocarpa. BMC Plant Biol. 14, 131. https://doi.org/10.1186/1471-2229-14-131.

Lin, E.P., Niu, M.Y., Huang, H.H., Li, X.Y., Zhang, J.H., Tong, Z.K., 2018. Molecular characterization of SQUAMOSA PROMOTER BINDING PROTEIN-LIKE (SPL) gene family in Betula luminifera. Front. Plant Sci. 9, 1-17. https://doi.org/10.3389/ fpls.2018.00608.

Mao, H.D., Yu, L.J., Li, Z.J., Yan, Y., Han, R., Liu, H., Ma, M., 2016. Genome-wide analysis of the SPL family transcription factors and their responses to abiotic stresses in maize. Plant Gene 6, 1-12. https://doi.org/10.1016/j.plgene.2016.03.003.

Matthews, C., Arshad, M., Hannoufa, A., 2019. Alfalfa response to heat stress is modulated by microRNA156. Physiol. Plant. 165, 830-842. https://doi.org/ 10.1111/ppl.12787.

Michaely, P., Bennett, V., 1992. The ANK repeat: a ubiquitous motif involved in macromolecular recognition. Trends Cell Biol. 2, 127-129. https://doi.org/10.1016/ 0962-8924(92)90084-Z. 
Miura, K., Ikeda, M., Matsubara, A., Song, X.J., Ito, M., Asano, K., Matsuoka, M., Kitano, H., Ashikari, M., 2010. OsSPL14 promotes panicle branching and higher grain productivity in rice. Nat. Genet. 42, 545-549. https://doi.org/10.1038/ ng. 592 .

Ning, K., Chen, S., Huang, H., Jiang, J., Yuan, H., Li, H., 2017. Molecular characterization and expression analysis of the SPL gene family with BpSPL9 transgenic lines found to confer tolerance to abiotic stress in Betula platyphylla Suk. Plant Cell Tissue Organ Cult. 130, 469-481. https://doi.org/10.1007/s11240-0171226-3.

Schmittgen, T.D., Livak, K.J., 2008. Analyzing real-time PCR data by the comparative CT method. Nat. Protoc. 3, 1101-1108. https://doi.org/10.1038/nprot.2008.73.

Shao, Y., Zhou, H.Z., Wu, Y., Zhang, H., Lin, J., Jiang, X., He, Q., Zhu, J., Li, Y., Yu, H., Mao, C., 2019. OsSPL3, an SBP-domain protein, regulates crown root development in rice. Plant Cell 31, 1257-1275. https://doi.org/10.1105/tpc.19.00038.

Shinde, H., Dudhate, A., Anand, L., Tsugama, D., Gupta, S.K., Liu, S., Takano, T., 2020. Small RNA sequencing reveals the role of pearl millet miRNAs and their targets in salinity stress responses. S. Afr. J. Bot. 132, 395-402. https://doi.org/10.1016/j. sajb.2020.06.011.

Si, L., Chen, J., Huang, X., Gong, H., Luo, J., Hou, Q., Zhou, T., Lu, T., Zhu, J., Shangguan, Y., Chen, E., Gong, C., Zhao, Q., Jing, Y., Zhao, Y., Li, Y., Cui, L., Fan, D., Lu, Y., Weng, Q., Wang, Y., Zhang, Q., Liu, K., Wei, X., An, K., An, G., Han, B., 2016. OsSPL13 controls grain size in cultivated rice. Nat. Genet. 48, 447-456. https://doi. org/10.1038/ng.3518.

Tang, M., Zhou, C., Meng, L., Mao, D., Peng, C., Zhu, Y., Huang, D., Tan, Z., Chen, C., Liu, C., Zhang, D., 2016. Overexpression of OSSPL9 enhances accumulation of $\mathrm{Cu}$ in rice grain and improves its digestibility and metabolism. J. Genet. Genomics 43, 673-676. https://doi.org/10.1016/j.jgg.2016.09.004.

Tian, F., Yang, D.C., Meng, Y.Q., Kong, L., Luo, J.C., Gao, G., 2017. PlantTFDB 4.0: toward a central hub for transcription factors and regulatory interactions in plants. Nucleic Acids Res. 45 (D1), D1040-D1045. https://doi.org/10.1093/nar/gkw982.

Tripathi, R.K., Goel, R., Kumari, S., Dahuja, A., 2017. Genomic organization, phylogenetic comparison, and expression profiles of the SPL family genes and their regulation in soybean. Dev. Genes Evol. 227, 101-119. https://doi.org/10.1007/ s00427-017-0574-7.

Varshney, R.K., Shi, C., Thudi, M., Mariac, C., Wallace, J., Qi, P., Zhang, H., Zhao, Y., Wang, X., Rathore, A., Srivastava, R.K., Chitikineni, A., Fan, G., Bajaj, P., Punnuri, S., Gupta, S.K., Wang, H., Jiang, Y., Couderc, M., Katta, M.A.V.S.K., Paudel, D.R., Mungra, K.D., Chen, W., Harris-Shultz, K.R., Garg, V., Desai, N., Doddamani, D., Kane, N.A., Conner, J.A., Ghatak, A., Chaturvedi, P., Subramaniam, S., Yadav, O.P., Berthouly-Salazar, C., Hamidou, F., Wang, Jianping, Liang, X., Clotault, J., Upadhyaya, H.D., Cubry, P., Rhoné, B., Gueye, M.C., Sunkar, R., Dupuy, C., Sparvoli, F., Cheng, S., Mahala, R.S., Singh, B., Yadav, R.S., Lyons, E., Datta, S.K., Hash, C.T., Devos, K.M., Buckler, E., Bennetzen, J.L., Paterson, A.H., Ozias-Akins, P., Grando, S., Wang, Jun, Mohapatra, T., Weckwerth, W., Reif, J.C., Liu, X., Vigouroux, Y., Xu, X., 2017. Pearl millet genome sequence provides a resource to improve agronomic traits in arid environments. Nat. Biotechnol. https://doi.org/ 10.1038/nbt.3943.

Wang, J.W., Czech, B., Weigel, D., 2009. miR156-regulated SPL transcription factors define an endogenous flowering pathway in Arabidopsis thaliana. Cell 138, 738-749. https://doi.org/10.1016/j.cell.2009.06.014.

Wang, P., Chen, D., Zheng, Y., Jin, S., Yang, J., Ye, N., 2018a. Identification and expression analyses of SBP-box genes reveal their involvement in abiotic stress and hormone response in tea plant (Camellia sinensis). Int. J. Mol. Sci. 19, 3404. https:// doi.org/10.3390/ijms19113404.

Wang, Q.L., Sun, A.Z., Chen, S.T., Chen, L.S., Guo, F.Q., 2018b. SPL6 represses signalling outputs of ER stress in control of panicle cell death in rice. Nat. Plants 4, 280-288. https://doi.org/10.1038/s41477-018-0131-z.

Wang, S., Wu, K., Yuan, Q., Liu, X., Liu, Z., Lin, X., Zeng, R., Zhu, H., Dong, G., Qian, Q., Zhang, G., Fu, X., 2012. Control of grain size, shape and quality by OsSPL16 in rice. Nat. Genet. 44, 950-954. https://doi.org/10.1038/ng.2327.

Xie, K., Wu, C., Xiong, L., 2006. Genomic organization, differential expression, and interaction of SQUAMOSA promoter-binding-like transcription factors and microRNA156 in rice. Plant Physiol. 142, 280-293. https://doi.org/10.1104/ pp.106.084475.

Xu, Y., Xu, H., Wall, M.M., Yang, J., 2020. Roles of transcription factor SQUAMOSA promoter binding protein-like gene family in papaya (Carica papaya) development and ripening. Genomics 112, 2734-2747. https://doi.org/10.1016/j. ygeno.2020.03.009.

Yamasaki, K., Kigawa, T., Inoue, M., Tateno, M., Yamasaki, T., Yabuki, T., Aoki, M., Seki, E., Matsuda, T., Nunokawa, E., Ishizuka, Y., Terada, T., Shirouzu, M., Osanai, T., Tanaka, A., Seki, M., Shinozaki, K., Yokoyama, S., 2004. A novel zincbinding motif revealed by solution structures of DNA-binding domains of Arabidopsis SBP-family transcription factors. J. Mol. Biol. 337, 49-63. https://doi. org/10.1016/j.jmb.2004.01.015.

Yu, N., Niu, Q.W., Ng, K.H., Chua, N.H., 2015. The role of miR156/SPLs modules in Arabidopsis lateral root development. Plant J. 83, 673-685. https://doi.org/ 10.1111/tpj.12919.

Yu, S., Galvão, V.C., Zhang, Y.C., Horrer, D., Zhang, T.Q., Hao, Y.H., Feng, Y.Q., Wang, S., Schmid, M., Wang, J.W., 2012. Gibberellin regulates the Arabidopsis floral transition through miR156-targeted SQUAMOSA PROMOTER BINDING-LIKE transcription factors. Plant Cell 24, 3320-3332. https://doi.org/10.1105/ tpc.112.101014.

Yuan, H., Qin, P., Hu, L., Zhan, S., Wang, S., Gao, P., Li, J., Jin, M., Xu, Z., Gao, Q., Du, A., Tu, B., Chen, W., Ma, B., Wang, Y., Li, S., 2019. OSSPL18 controls grain weight and grain number in rice. J. Genet. Genomics 46, 41-51. https://doi.org/10.1016/j. jgg.2019.01.003.

Yue, E., Li, C., Li, Y., Liu, Z., Xu, J.H., 2017. MiR529a modulates panicle architecture through regulating SQUAMOSA PROMOTER BINDING-LIKE genes in rice (Oryza sativa). Plant Mol. Biol. 94, 469-480. https://doi.org/10.1007/s11103-017-0618-4.

Zhang, H.X., Jin, J.H., He, Y.M., Lu, B.Y., Li, D.W., Chai, W.G., Khan, A., Gong, Z.H., 2016. Genome-wide identification and analysis of the SBP-box family genes under phytophthora capsici stress in pepper (Capsicum annuum L.). Front. Plant Sci. 7, 1-14. https://doi.org/10.3389/fpls.2016.00504.

Zhang, S.D., Ling, L.Z., 2018. Diversification of SQUANOSA promoter binding proteinlike (SPL) genes by changes of miR156/529 binding sites in land plants. Plant Gene 14, 55-63. https://doi.org/10.1016/j.plgene.2018.04.007.

Zhang, X., Dou, L., Pang, C., Song, M., Wei, H., Fan, S., Wang, C., Yu, S., 2014. Genomic organization, differential expression, and functional analysis of the SPL gene family in Gossypium hirsutum. Mol. Gen. Genomics. 290, 115-126. https://doi.org/10.1007/ s00438-014-0901-x.

Zhou, M., Tang, W., 2019. MicroRNA156 amplifies transcription factor-associated cold stress tolerance in plant cells. Mol. Gen. Genomics. 294, 379-393. https://doi.org/ 10.1007/s00438-018-1516-4. 